\title{
Immune marker levels in severe mental disorders: associations with polygenic risk scores of related mental phenotypes and psoriasis
}

\author{
Maren Caroline Frogner Werner $\mathbb{D}^{1 凶}$, Katrine Verena Wirgenes ${ }^{1,2}$, Alexey Shadrin ${ }^{1}$, Synve Hoffart Lunding ${ }^{1}$, Linn Rødevand ${ }^{1}$, \\ Gabriela Hjell (iD) ${ }^{1,3}$, Monica Bettina Elkjær Greenwood Ormerod (D) ${ }^{1}$, Marit Haram ${ }^{1}$, Ingrid Agartz ${ }^{4,5,6}$, Srdjan Djurovic (iD ${ }^{2,7}$, \\ Ingrid Melle $\mathbb{D}^{1}$, Pål Aukrust ${ }^{8,9,10}$, Thor Ueland ${ }^{8,9,11}$, Ole Andreas Andreassen (iD) ${ }^{1}$ and Nils Eiel Steen (iD ${ }^{1}$
}

(c) The Author(s) 2022

Several lines of evidence implicate immune abnormalities in the pathophysiology of severe mental disorders (SMD) and comorbid mental disorders. Here, we use the data from genome-wide association studies (GWAS) of autoimmune diseases and mental phenotypes associated with SMD to disentangle genetic susceptibilities of immune abnormalities in SMD. We included 1004 patients with SMD and 947 healthy controls (HC) and measured plasma levels of IL-1Ra, sIL-2R, gP130, sTNFR-1, IL-18, APRIL, and ICAM-1. Polygenic risk scores (PRS) of six autoimmune disorders, CRP, and 10 SMD-related mental phenotypes were calculated from GWAS. General linear models were applied to assess the association of PRS with immune marker abnormalities. We found negative associations between PRS of educational attainment and IL-1Ra $(P=0.01)$ and IL-18 $(P=0.01)$. There were nominal positive associ ations between PRS of psoriasis and $\operatorname{sgp} 130(P=0.02)$ and PRS of anxiety and IL-18 $(P=0.03)$, and nominal negative associations between PRS of anxiety and SIL-2R ( $P=0.02)$ and PRS of educational attainment and SIL-2R $(P=0.03)$. Associations explained minor amounts of the immune marker plasma-level difference between SMD and HC. Different PRS and immune marker associations in the SMD group compared to HC were shown for PRS of extraversion and IL-1Ra ([interaction effect (IE), $P=0.002$ ), and nominally for PRS of openness and IL-1Ra (IE, $P=0.02$ ) and sTNFR-1 (IE, $P=0.04)$. Our findings indicate polygenic susceptibilities to immune abnormalities in SMD involving genetic overlap with SMD-related mental phenotypes and psoriasis. Associations might suggest immune genetic factors of SMD subgroups characterized by autoimmune or specific mental features.

Translational Psychiatry (2022)12:38; https://doi.org/10.1038/s41398-022-01811-6

\section{INTRODUCTION}

Bipolar disorder (BD) and schizophrenia (SCZ) are among the leading causes of disability worldwide. The pathophysiology of these severe mental disorders (SMD) is not fully understood. Their estimated heritability is $60-80 \%$ and recent genome-wide association studies (GWASs) have revealed a large number of common genetic risk variants [1, 2] with extensive overlap between the disorders [3]. Moreover, evidence suggests extensive genetic overlap with several associated traits [4] and comorbid disorders [1, 5]. The growing body of GWAS can be used to generate polygenetic risk scores (PRS) of SMD-related phenotypes to study core features of SMD [6].

Involvement of the immune system in SMD is supported by several lines of evidence [7, 8]. Genetic loci in immune-related regions are associated with $\mathrm{SCZ}$ and $\mathrm{BD}$ [9] and infections during pregnancy, potentially inducing immune activation, increase the child's risk of developing SCZ and BD decades later [10]. Similarly, large registry studies have identified an increased risk associated with prior infections [11, 12]. Inflammation seems to modulate brain mechanisms underlying clinical characteristics of SMD [13]. Moreover, similar signs of systemic low-grade immune activation and inflammation are found in SCZ and BD [14] and associations with symptoms [15] and pharmacological treatment response [16] are reported. However, the causative mechanisms of immune abnormalities, as well as the pathogenic importance of the different pathways that are upregulated, are unknown.

Autoimmune disease is characterized by immune-mediated attacks by autoantibodies and self-reactive lymphocytes in particular T cells and is a known comorbidity of SMD [17]. There is evidence of shared genetic risk with SMD [9] and neuropsychiatric symptoms in autoimmune diseases [17]. While the immune signaling system is extensive and autoimmune diseases are associated with different circulating immune markers [18], some of these markers are also linked to SMD, suggesting not only

\footnotetext{
${ }^{1}$ NORMENT, Division of Mental Health and Addiction, Oslo University Hospital \& Institute of Clinical Medicine, University of Oslo, Oslo, Norway. ${ }^{2}$ Department of Medical Genetics, Oslo University Hospital, Oslo, Norway. ${ }^{3}$ Department of Psychiatry, Ostfold Hospital, Graalum, Norway. ${ }^{4}$ NORMENT, Institute of Clinical Medicine, University of Oslo, Oslo, Norway. ${ }^{5}$ Department of Psychiatric Research, Diakonhjemmet Hospital, Oslo, Norway. ${ }^{6}$ Department of Clinical Neuroscience, Centre for Psychiatric Research, Karolinska Institutet, Stockholm, Sweden. ${ }^{7}$ NORMENT, Department of Clinical Science, University of Bergen, Bergen, Norway. ${ }^{8}$ Research Institute of Internal Medicine, Oslo University Hospital, Rikshospitalet, Oslo, Norway. ${ }^{9}$ Faculty of Medicine, University of Oslo, Oslo, Norway. ${ }^{10}$ Section of Clinical Immunology and Infectious Diseases, Oslo University Hospital Rikshospitalet, Oslo, Norway. ${ }^{11}$ K.G. Jebsen - Thrombosis Research and Expertise Center (TREC), University of Tromsø, Tromsø, Norway. ${ }^{凶}$ email: m.c.f.werner@medisin.uio.no
}

Received: 23 September 2021 Revised: 22 December 2021 Accepted: 10 January 2022

Published online: 26 January 2022 
shared genetic risk factors, but also shared immune-related mechanisms. Such markers include interleukin-18 (IL-18) and interleukin-1 receptor antagonist (IL-1Ra) implicated in among others rheumatoid arthritis (RA), a proliferating-inducing ligand (APRIL) involved in RA and systemic lupus erythematosus (SLE), interleukin-6 (IL-6) in RA and inflammatory bowel disease (IBD) and soluble interleukin-2 receptor (sIL-2R) as a marker of T-cell activation involved in a wide range of autoimmune disorders like IBD and SLE [19-22].

Several mental traits and disorders are genetically associated with SMD [23]. Such examples of mental disorders include major depressive disorder (MDD), anxiety disorders, and autism spectrum disorders (ASD) [24-27]. Further, the genetic association between SMD and different mental traits include cognitive abilities [28], a core impairment of SMD and personality traits of openness, extraversion, and neuroticism [29, 30]. The immune system is also suggested to be involved in these SMD-related disorders and traits. Comorbid mental disorders are associated with immune marker abnormalities [31-34] and immune-related genetic factors $[9,35,36]$. Cognitive abilities correlate with immune factors [37] and immune mechanisms in personality traits are indicated [38].

Physiological levels of immune markers are in addition to a range of non-genetic factors, influenced by complex genetics [39]. Thus, mapping the genetic architecture of immune abnormalities in SMD may provide novel pathophysiological knowledge. Applying multiple PRS of SMD-related phenotypes for explaining characteristics within SMD was recently suggested [4]. Here, we tested the hypothesis that PRS of autoimmune or SMD-related mental phenotypes with immune associations can explain detectable, but small amounts [40] of immune marker abnormalities in SMD. We applied PRS of autoimmune diseases and mental phenotypes, including mental disorders [31-33, 41], cognitive phenotypes [37, 42], and personality traits [38] in a naturalistic sample of SMD to investigate associations with immune markers. SCZ and BD spectrum-specific analyses were further explored.

\section{METHODS \\ Study setting}

The study is a part of the Thematically Organized Psychosis Study (TOP) at the Norwegian Centre for Mental Disorders Research (NORMENT). The TOP study includes patients from the major hospitals in the Oslo region with a diagnosis of a SCZ spectrum (schizophrenia, schizophreniform disorder, schizoaffective disorder, delusional disorder, brief psychotic disorder, and psychosis not otherwise specified (NOS), from here termed "SCZ") or BD spectrum (bipolar 1 disorder, bipolar 2 disorder, bipolar disorder NOS, and major depressive disorder with psychotic features, from here termed "BD") disorder as the main inclusion criteria. Participants must be able to provide written informed consent and be between the ages of 18-65. Healthy controls $(\mathrm{HC})$ are randomly selected from statistical records from the same catchment areas as the patients. $\mathrm{HC}$ are included based on no history of severe mental illness, illicit drug abuse or dependency, or close relatives with severe mental illness. Both patients and $\mathrm{HC}$ are excluded based on somatic conditions interfering with brain function, neurological disorder, IQ $<70$, or history of severe head trauma. In this study, we additionally applied the following exclusion criteria: data of participants other than European ethnicity were not included in the analyses to avoid issues with population stratification, and participants with CRP level of 10 or above $(N=114)$ were excluded to remove potential effects of acute infection on the immune markers. The resulting sample with immune measures included $N=1004$ patients with SMD and $N=947 \mathrm{HCs}$ (Table 1), out of which PRS data was available for $N=1802$ individuals (Table 2). Immune measures from the current sample have been reported in overlapping samples [43-47].

\section{Clinical assessment}

Diagnostics are made by use of the Structured Clinical Interview (SCID-1) for the Diagnostic and Statistical Manual of Mental Disorders (DSM)-IV.

Table 1. Demographics and clinical data.

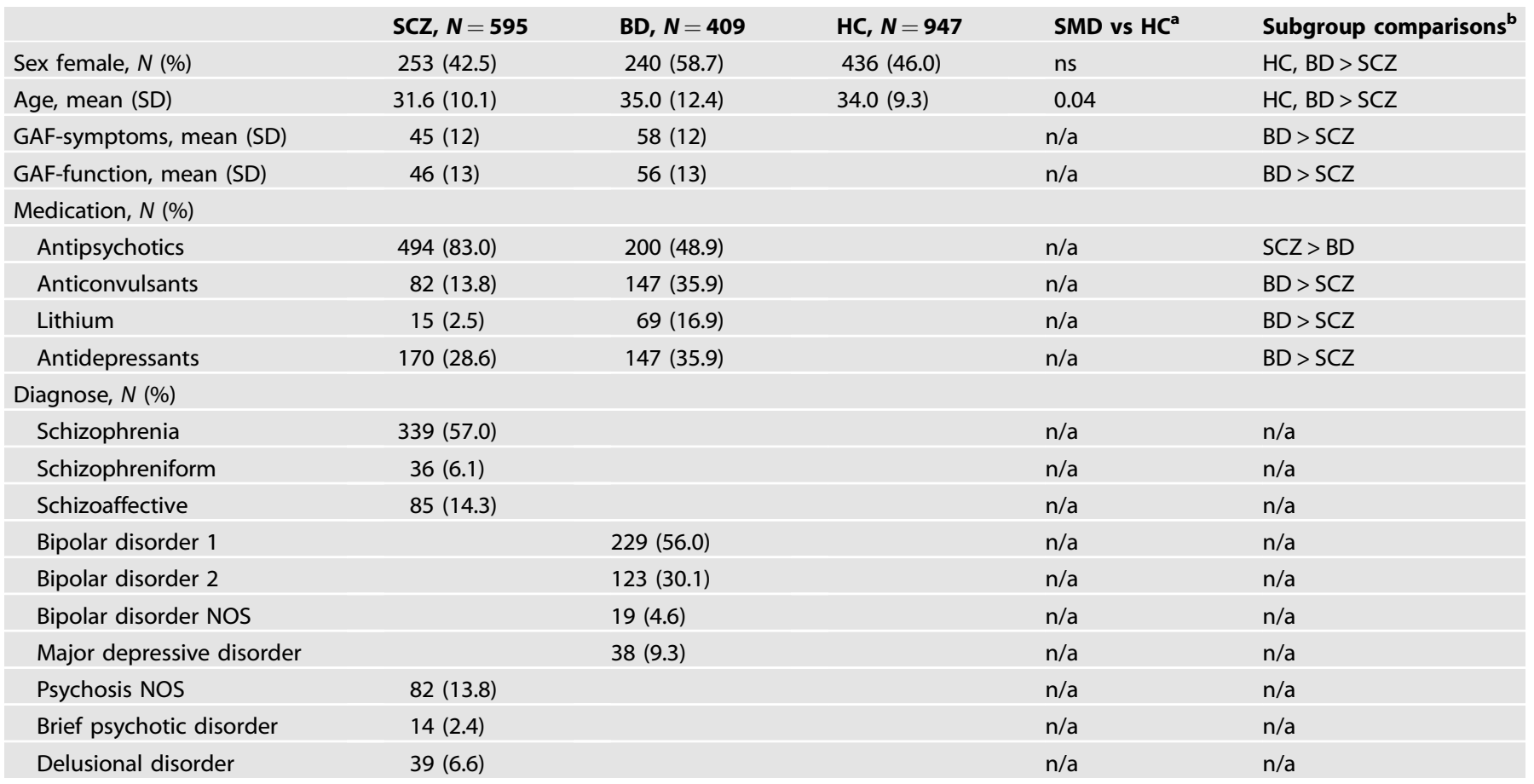

$B D$ bipolar spectrum disorders, GAF general assessment of functioning scale, $n$ s not significant, $n / a$ not applicable, SCZ schizophrenia spectrum disorders, SMD severe mental disorders.

${ }^{a} P$ value of SMD versus HC: chi square for categorical variables, $t$ test for continuous variables, ${ }^{\text {b }}$ chi square for categorical variables, ANOVA for continuous variables. 
Table 2. Polygenic risk scores.

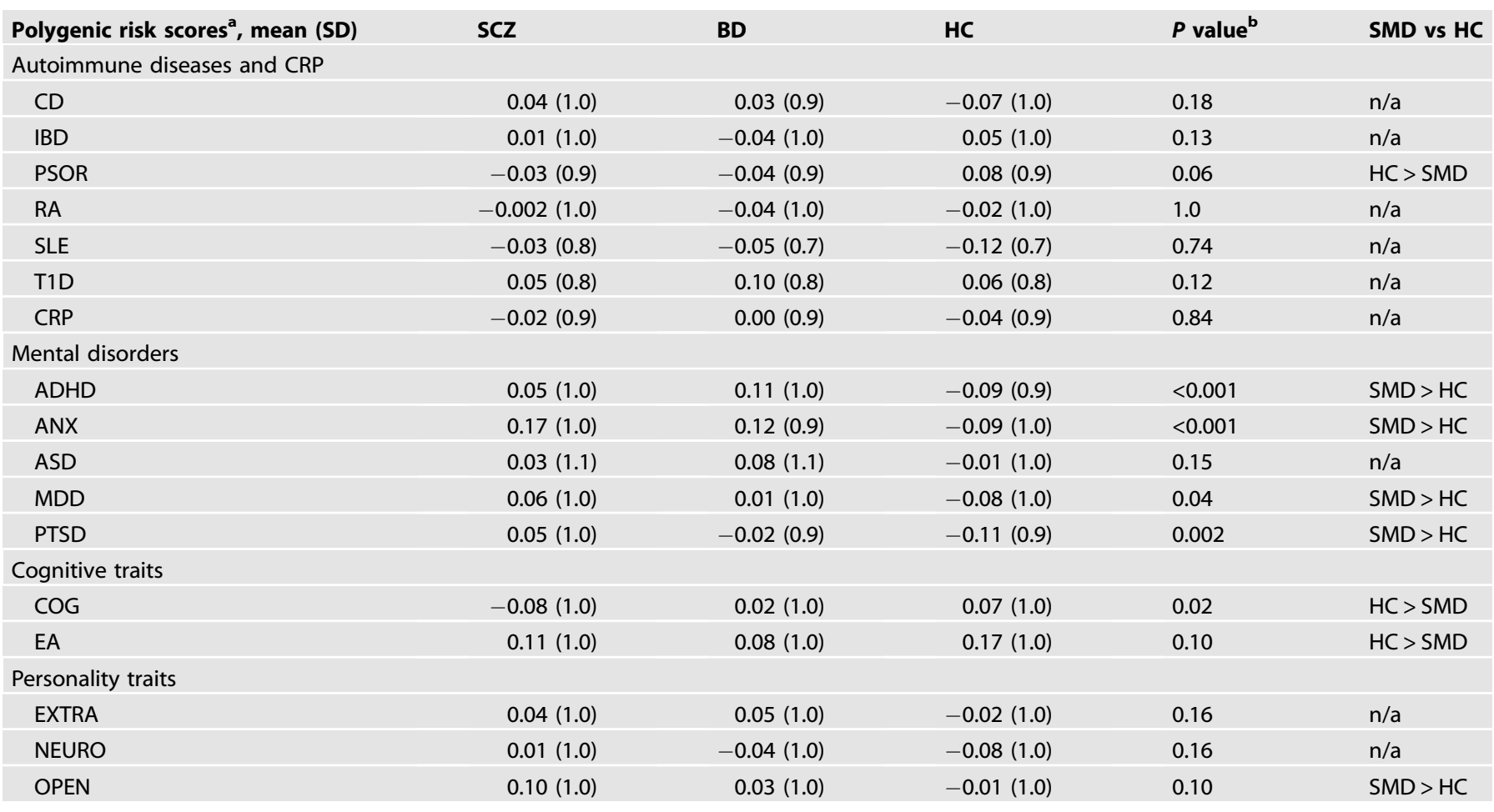

$A D H D$ attention-deficit hyperactivity disorder, $A N X$ anxiety, $A S D$ autism spectrum disorder, $B D$ bipolar spectrum disorders, CD Crohn's disease, COG cognition, CRP C-reactive protein, EA educational attainment, EXTRA extraversion, $H C$ healthy controls, IBD inflammatory bowel disease, MDD major depressive disorder, $n /$ a not applicable, NEURO neuroticism, OPEN openness, PC principal component, PSOR psoriasis, PTSD post-traumatic stress disorder, RA rheumatoid arthritis, SCZ schizophrenia spectrum disorders, SD standard deviation, SMD severe mental disorders (SCZ and BD), SLE systemic lupus erythematosus, T1D type 1 diabetes. ${ }^{a}$ Standardized values.

${ }^{\mathrm{b}} P$ value of SMD vs HC: ANCOVA with adjustments for PC1, PC2, and genetic batch.

Diagnostic interviews are performed by psychologists and physicians supervised by a clinical professor in psychiatry. The research personnel is all comprehensively trained for the interviews with a UCLA training program [48].

\section{Polygenic risk scores}

DNA was extracted from blood and saliva samples collected at the time of inclusion of the participants. Samples were genotyped at deCODE Genetics (Reykjavik, Iceland) using Illumina Human OmniExpress-12, OmniExpress24, and Global Screening Array chips. Pre-imputation quality control was performed using PLINK 1.9. Briefly, variants were excluded if they had a low genotyping rate $(<95 \%)$, deviated from Hardy-Weinberg equilibrium $(P<$ $10^{-4}$ ), or occurred at significantly different frequencies in different genotyping batches (FDR $<0.5)$. Whole-individual genotypes were excluded if they had low coverage $(<80 \%)$, a high likelihood of contamination (heterozygosity above mean +5 standard deviations) or mismatch between genetic and reported sex. The quality-controlled genotypes were phased using Eagle [49], and missing variants were then imputed with $\mathrm{MaCH}[50,51]$ using haplotype reference consortium (HRC) trans-ethnic reference panel (version 1.1) [52]. Following the quality control and imputation procedure, variants with information score $<0.8$ or minor allele frequency $<1 \%$ were removed. In addition, individual genotypes imputed with less than $75 \%$ confidence were set to missing, and the remaining ones were converted to best guess hard allelic dosages. PRS were computed following the method described by Purcell et al. [53] using PRSice-2 [54].

Our study included PRS of phenotypes with detected genetic associations to SMD and immune links, including (1) the autoimmune diseases rheumatoid arthritis (PRS-RA) [55], systemic lupus erythematosus (PRS-SLE) [56], inflammatory bowel disease (PRS-IBD) [57], psoriasis (PRSPSOR) [58], celiac disease (PRS-CD) [59], type 1 diabetes (PRS-T1D) [60], as well as C-reactive protein (PRS-CRP) [61], (2) the mental disorders major depressive disorder (PRS-MDD) [62], anxiety (PRS-ANX) [63], autism spectrum disorders (PRS-ASD) [64], attention-deficit hyperactivity disorder
(PRS-ADHD) [65], post-traumatic stress disorder (PRS-PTSD) [66], (3) the cognitive traits general intelligence (PRS-COG) [67] and the cognitive ability proxy educational attainment (PRS-EA) [68], and (4) the personality traits neuroticism (PRS-NEURO), openness (PRS-OPEN) and extraversion (PRS-EXTRA) [30]. We used the $P$ value threshold of 0.05 for the selection of single-nucleotide polymorphisms (SNPs) in the PRS analyses. All PRS were standardized before analysis. See Supplementary Table 1 for a summary of the GWASs used for the calculation of PRS in our study.

\section{Immune markers}

Immune markers were selected based on previously documented associations to SMD or neuropsychiatric symptomatology [14, 69-71], comprising interleukin-1 receptor antagonist (IL-1Ra), soluble interleukin-2 receptor (sIL2R), interleukin-18 (IL-18), soluble glycoprotein 130 (sgp130), a proliferationinducing ligand (APRIL), tumor necrosis factor receptor 1 (TNFR-1) and intercellular cell adhesion molecule 1 (ICAM-1). Following the same methods as described by Mørch et al. [43], plasma levels of the immune markers were measured in duplicate by enzyme immunoassays (EIA) applying commercially available antibodies (R\&D Systems, Minneapolis, MN, USA) in a 384 format using a combination of a SELMA (Jena, Germany) pipetting robot and a BioTek (Winooski, VT, USA) dispenser/washer. Absorption was read at $450 \mathrm{~nm}$ with wavelength correction set to $540 \mathrm{~nm}$ using an ELISA plate reader (Bio-Rad, Hercules, CA, USA). Intra- and inter-assay coefficients of variation were $<10 \%$ for all ElAs. For the immunoassays, blood was sampled between 8:00 and 17:00 using EDTA vials and the plasma was isolated within the next working day and stored at $-80^{\circ} \mathrm{C}$. Plasma collection was performed subsequently as participants were included in the study, and "freezer storage time" (years) was recorded and included as a continuous independent variable in the main statistical model.

\section{Statistical analyses}

All statistical analyses were performed using the Statistical Package for the Social Sciences (SPSS Inc., Chicago II, version 26). Histograms, Q-Q plots and 
Kolmogorov-Smirnov statistics were used to assess distributions of data. For group comparison of demographic and clinical data, we used $t$ test and ANOVA for continuous variables and Chi-Square test for categorical variables. Pearson's $r$ or Spearman's rho were used for correlation analyses. The main analyses were performed with Analysis of Covariance (ANCOVA), with immune markers as dependent variables. Immune marker distributions were carefully assessed, and all markers were logarithmically transformed with standardized removal of residuals more than $3 \times$ interquartile range (IQR) or $1.5 \times$ IQR below and above the first and third quartile, respectively, depending on the degree of deviation (3 IQR for gp130, sTNFR-1, IL-18, and ICAM-1 and $1.5 \mathrm{IQR}$ for IL-1Ra, sIL-2R, and APRIL). First, we identified PRS and immune marker variables with indicated $(P \leq 0.1)$ differences between SMD and HC (Table 2 and Supplementary Table 2) and bivariate correlations (in SMD, Supplementary Table 3). We then performed the main analyses with these variables comparing immune marker differences between SMD and HC ("diagnosis variable") with and without adjustments for PRS to assess the effect of PRS on immune marker differences. Moreover, all PRS correlating with immune markers in SMD were tested for interaction effects with the diagnosis variable. Main analyses, including interactions, were repeated in subsamples by substituting SMD with SCZ ("subsample SCZ-HC") and BD ("subsample BD-HC"), respectively. Main analyses were adjusted for age at blood sampling, sex, freezer storage time, genetic principal components 1 and 2 and genetic batch by including these as independent variables in the ANCOVA. Based on established genetic overlap with SMD and immune links of SMD-related phenotypes, we applied a moderate correction of the significance level of $P=0.0125(0.05 / 4)$ to correct for testing of PRS of the four groups of autoimmune disorders, mental disorders, cognitive traits, and personality traits.

\section{RESULTS}

\section{Demographic data and immune markers}

The mean age was lower in SMD than in $\mathrm{HC}(P=0.04)$ with younger participants in $\mathrm{SCZ}$ than in the $\mathrm{BD}$ and $\mathrm{HC}$ (both $P<$ $0.001)$. The proportion of females was lowest in $S C Z$ and highest in $\mathrm{BD}$ (all $P<0.001$ ), see Table 1. Plasma levels of all immune markers differed significantly between SMD and HC (Supplementary Table 2); however, case-control differences are more specifically reported in other papers from our group [43-47] [submitted]. Bivariate correlation analyses of PRS and immune marker levels in SMD indicated three correlations with PRS of autoimmune diseases ("PRS-Autoimm") and seventeen for PRS of mental disorders, traits, or cognitive abilities ("PRS-Ment"). Of these, there were one PRSAutoimm (i.e., PRS-PSOR) and seven PRS-Ment (i.e., PRS-ANX, PRSMDD, PRS-ADHD, PRS-PTSD, PRS-OPEN, PRS-COG, and PRS-EA) with the indicated difference between SMD and HC. Details are found in Table 2 and Supplementary Table 3.

\section{Immune marker levels: main effects of PRS}

PRS-Autoimm: PRS-PSOR was nominally positively associated with plasma levels of $\operatorname{sgp} 130(P=0.02)$; the plasma-level difference between SMD and HC decreased by 0.2 percentage points when adding PRS-PSOR to the model.

PRS-Ment: PRS-ANX was nominally negatively associated with sIL-2R $(P=0.02)$ and positively with IL-18 $(P=0.03)$; the plasmalevel difference between SMD and HC of SIL-2R and IL-18 increased by 0.6 percentage points and decreased by 2.0 percentage points, respectively, when adding PRS-ANX to the models. PRS-EA was negatively associated with IL-1Ra $(P=0.01)$ and IL-18 $(P=0.01)$, and at nominal level with sIL-2R $(P=0.03)$; the plasma-level difference of IL-1Ra (18.6\%) and sIL-2R (13.2\%) were unchanged when adding PRS-EA to the models, whereas the plasma-level difference of IL-18 decreased by 0.7 percentage points. See Table 3 for statistical details of PRS main effects. There were no other significant main effects of PRS in the main analyses.

\section{Immune marker levels: interaction effects of PRS}

PRS-Autoimm: There were no significant interaction effects between PRS and the diagnosis variable.

PRS-Ment: Nominal SMD specific negative associations was found for PRS-EXTRA and IL-1Ra $(P=0.02$ [interaction effect, $P=$ 0.002 ]) and for PRS-OPEN and IL-1Ra $(P=0.02$ [interaction effect,

Table 3. Main effects of polygenic risk scores on immune markers ${ }^{a}$.

\begin{tabular}{|c|c|c|c|c|c|c|}
\hline & Immune marker & df & F-ratio & $P$ value & $n_{p}^{2}$ & PP change ${ }^{c}$ \\
\hline \multicolumn{7}{|l|}{ Total sample } \\
\hline PRS-PSOR & sgp130 & 1,1118 & 5.86 & 0.02 & 0.005 & -0.2 \\
\hline \multirow[t]{2}{*}{ PRS-ANX } & sIL-2R & 1,1050 & 5.19 & 0.02 & 0.005 & 0.6 \\
\hline & IL-18 & 1,1520 & 4.77 & 0.03 & 0.003 & -2.0 \\
\hline \multirow[t]{2}{*}{ PRS-EA } & IL-1Ra & 1,1087 & 6.46 & 0.01 & 0.006 & 0.0 \\
\hline & sIL-2R & 1,1050 & 4.79 & 0.03 & 0.005 & 0.0 \\
\hline \multicolumn{7}{|c|}{ Subsample SCZ-HC } \\
\hline PRS-PSOR & sgp130 & 1,885 & 4.95 & 0.03 & 0.006 & -0.2 \\
\hline PRS-ANX & IL-18 & 1,1196 & 3.92 & 0.05 & 0.003 & -2.1 \\
\hline \multirow[t]{2}{*}{ PRS-EA } & IL-1Ra & 1,861 & 4.09 & 0.04 & 0.005 & 0.4 \\
\hline & IL-18 & 1,1196 & 5.89 & 0.02 & 0.005 & -0.4 \\
\hline
\end{tabular}

$A N X$ anxiety, $B D$ bipolar spectrum disorders, $d f$ degrees freedom, $E A$ educational attainment, $I L-18$ interleukin- $18, I L-1 R a$ interleukin- 1 receptor antagonist, $\eta_{p}{ }^{2}$ partial eta squared, $P P$ percentage points, $P R S$ polygenic risk score, $P S O R$ psoriasis, SCZ schizophrenia spectrum disorders, s/L-2R soluble interleukin-2 receptor, sgp130 soluble glycoprotein 130 .

andependent variables: PRS, age, sex, freezer storage time, patients vs healthy controls, genetic principal components 1 and 2 and genetic batch; dependent variables: immune markers.

${ }^{b}$ Subsample SCZ-HC: participants with schizophrenia spectrum disorders and healthy controls; subsample BD-HC: participants with bipolar spectrum disorders and healthy controls.

${ }^{C}$ Percentage points change in immune marker plasma-level difference between diagnostic subgroups and healthy controls by adding PRS to the statistical model. 
$P=0.02])$ and sTNFR-1 $(P=0.04$ [interaction effect, $P=0.04$ ]). See Supplementary Fig. 1. There were no other significant interaction effects between PRS and the diagnosis variable.

\section{Subsample analysis}

For separate analyses of SCZ-HC and BD-HC subsamples, see Table 3 and Supplementary Results. In general, subsample findings corresponded with the main findings. The association between PRS-EA and IL-1Ra in the BD-HC subsample survived correction for multiple testing $(P=0.01)$ and in addition there was a SCZ-specific association for PRS-EA and ICAM-1 $(P=0.003$, [interaction effect, $P=0.01])$. Levene's test was significant in ANCOVA analysis of STNFR-1 and in several analyses of IL-18.

\section{DISCUSSION}

The main findings of the study were significant associations of PRS-EA, and nominal associations for PRS-PSOR and PRS-ANX, with immune marker levels across patients with SMD and HC. Nominal associations that were specific for patients of immune levels and PRS-EXTRA and PRS-OPEN were detected by interaction analysis. Despite the limited explanatory power of PRS, the large sample allowed well-adjusted analyses indicating associations of genetic risk for autoimmune diseases and in particular for mental phenotypes with components of low-grade inflammation and immune activation in SMD; however, the correlations constituted small changes in immune marker differences between patients and HC. The involvement of several PRS-Ment might indicate effects on immune activation related to specific phenotypic features within SMD.

PRS-EA was negatively associated with IL-1Ra and IL-18 across SMD and HC, and both associations were confirmed in the SCZ-HC and $\mathrm{BD}-\mathrm{HC}$ subsamples. We used educational attainment as a proxy for cognitive ability based on known associations [72], including genetic correlation [73]. Importantly, the clinical relevance of our findings is substantiated by reports of cognitive correlations in patient groups. In particular, elevated IL-1Ra is found to be associated with poorer cognition in BD [74] and IL-18 is associated with cognition although with mixed directions in Alzheimer's disease and SCZ $[75,76]$. Moreover, as IL-1Ra is regarded as a marker of IL- $1 \mathrm{~b}$ activity, the combined effects of IL$1 \mathrm{Ra}$ and IL-18 could implicate the involvement of Nod-like receptor (NLR) family, pyrin domain-containing 3 (NLRP3) inflammasomes, the major source of both IL-1 and IL-18. Interestingly, NLRP3 inflammasomes have recently been suggested to link psychological stress, depression, and systemic illness [77] and have been linked to severe mental illness [78]. An interaction effect in the subsample analysis suggested a SCZ-specific negative relationship of PRS-EA with ICAM-1 (Supplementary Results). Increased ICAM-1 with decreased general cognitive abilities is found in older people [79]. Similarly, a previous PRS study support of a role for cell adhesion molecules (CAMs) in cognitive function in SCZ [80]. Thus, our findings indicate a polygenic interplay regulating cytokines involved in the cognitive symptom dimension of SMD, potentially explaining immune abnormalities in subgroups with cognitive impairments.

In general, there were few associations with PRS-Autoimm and the examined inflammatory markers. However, our findings suggest an association between PRS-PSOR and sgp130 abnormalities in SMD. Psoriasis is an autoimmune disease with modest systemic aberrations of IL-6, CRP, TNF- $a$, E-selection, and ICAM [81] and comorbidity with SCZ [82]. Gp130 is the common signal transducer in the IL- 6 cytokine family and reflect the activation of several of these cytokines. The positive correlation is also supported by elevated expression of gp130 in patients with psoriasis [83]. Although a minor impact on the difference between SMD and HC, the current association of sgp130 might reflect the genetic risk of sgp130 abnormalities in autoimmune-related subgroups of SMD, in line with the broad comorbidity of autoimmune diseases with SMD [17]. The trend-level lower PRSPSOR in SMD versus HC is at odds with a recently indicated small positive genetic correlation between SCZ and psoriasis [84]. The difference might be related to the current use of another and more recent GWAS of psoriasis. However, the finding suggests a potential of leveraging genetics of autoimmune diseases in elucidating immune mechanisms in SMD with the increasing power of GWAS.

Associations of PRS-ANX with IL-18 and sIL-2R were also indicated. Anxiety is genetically correlated to [3] and a common comorbidity of SCZ. The comorbidity exists also in prodromal stages [85], suggesting associations beyond overt psychotic symptoms. PRS-ANX was positively associated with IL-18 across SMD and HC, as well as in SCZ. The positive link with IL-18 is further supported by elevated levels of IL-18 in anxiety including increased levels with severity, indicating clinical implications [86]. While PRS-ANX was negatively associated with sIL-2R across SMD and $\mathrm{HC}$, subgroup analyses also suggested a BD-specific negative association not detected in the overall sample. Others have reported decreased levels of sIL-2R in anxiety [87], thus supporting the current negative correlation. However, sIL-2R may vary with clinical state in BD [88]. Thus, the interaction between PRS-ANX and $B D$ suggest an interplay with other susceptibilities in sIL-2R regulation related to clinical characteristics. Moreover, the positive association of PRS-ANX with IL-18 and a negative association with sIL-2R as robust marker of T-cell activation could implicate that innate and adaptive immunity may differently affect anxiety.

In addition to the main effects of PRS of autoimmune and SMDrelated mental phenotypes, PRS of personality traits were related to immune markers, particularly IL-1Ra, in interactions with SMD. The findings are in line with the documented association of personality traits with immune aberrations [38]. Decreased PRS-OPEN and PRSEXTRA were both associated with increased IL-1Ra in SMD and SCZ, similar to the correlation between PRS-OPEN and STNFR-1 in SMD. Interestingly, both openness and SCZ may be linked to dopamine activity in substantia nigra [89] and creativity [90] and a negative association with psychotic experiences was recently shown in a nonclinical sample [91]. The effects correspond with evidence suggesting widespread interactions in immune mechanisms [92]. Although speculative, one might hypothesize a mechanism involving the hypothalamic-pituitary-adrenal (HPA) axis given its association with immune responses and dysregulation in SMD [93, 94]. The interactions support complex associations underlying immune mechanisms also in psychiatric disorders, in line with previous studies [95]. Moreover, common immune pathways linking personality traits and SMD symptomatology may be hypothesized.

The strengths of this study are large, well-characterized sample of participants and a selection of immune markers robustly associated with SMD. The inclusion of SMD-related mental PRS in combination with the immune markers is to our knowledge the first of its kind. Limitations include a cross-sectional format that makes us unable to assess the stability of the associations. Also, the repeated testing increases risk of type 1 error; however, the analyses were based on known immune differences between SMD and $\mathrm{HC}$ and all PRS phenotypes were selected based on genetic associations to SMD and associations to immune markers. Moreover, given the lack of studies of underlying mechanisms of systemic inflammation in SMD, a stricter correction for multiple testing may lead to ignoring hypotheses that merit further investigation. Still, the moderate correction for multiple testing and significant Levene's test of some of the main ANCOVAs, warrants cautious interpretation of the results. Moreover, plasma was isolated and frozen within the next working day, as immediate freezing was not feasible. Although conflicting evidence [96] studies suggest reasonable stability of immune markers under various conditions [97]. Thus, while bias from potential blood handling issues or other non-specific effects 
cannot be fully excluded, a significant impact on the results seems less likely in the current setting of well-adjusted analyses and a large sample size. Moreover, major influence by unidentified factors is further prevented by the careful removal of outliers from the immune markers. The main associations explained small amounts of the difference in immune marker plasma level between SMD and HC. This is in line with findings in comparable studies [40]. The limited explanatory power is not unexpected given the complexity of SMD mechanisms, the impact of nongenetic influences on the immune system including stress responses related to clinical state [98], high level of polygenicity with potential interaction effects and missing heritability of the phenotypes $[68,99]$ and uncertain heritability of immune components [100].

In conclusion, we found associations of PRS of immune-linked SMD-related mental phenotypes and nominally of psoriasis with immune markers in SMD and HC. This suggests that genetic susceptibility of these phenotypes might be involved in mechanisms underlying systemic immune abnormalities often seen in SMD, which seems to be partly disease-specific. Although representing small changes, our findings indicate genetic susceptibilities in SMD immune dysregulation for further investigation, potentially of specific interest in subgroups based on autoimmune or mental characteristics, which could be of future relevance in personalized intervention studies. Moreover, the involvement of IL-1Ra and IL-18 in the detected association with various PRS may underscore a particular role for innate immunity in SMD.

\section{REFERENCES}

1. Smeland $O B$, Frei O, Dale AM, Andreassen OA. The polygenic architecture of schizophrenia-rethinking pathogenesis and nosology. Nat Rev Neurol. 2020;16:366-79.

2. Mullins N, Forstner AJ, O'Connell KS, Coombes B, Coleman JRI, Qiao Z, et al. Genome-wide association study of more than 40,000 bipolar disorder cases provides new insights into the underlying biology. Nat Genet. 2021;53:817-29.

3. Brainstorm Consortium, Anttila V, Bulik-Sullivan B, Finucane HK, Walters RK, Bras $\mathrm{J}$, et al. Analysis of shared heritability in common disorders of the brain. Science. 2018;360:eaap8757.

4. Dickinson D, Zaidman SR, Giangrande EJ, Eisenberg DP, Gregory MD, Berman KF. Distinct polygenic score profiles in schizophrenia subgroups with different trajectories of cognitive development. Am J Psychiatry. 2020;177:298-307.

5. Bahrami S, Steen NE, Shadrin A, O'Connell K, Frei O, Bettella F, et al. Shared genetic loci between body mass index and major psychiatric disorders: a genome-wide association study. JAMA Psychiatry. 2020;77:503-12.

6. Murray GK, Lin T, Austin J, McGrath JJ, Hickie IB, Wray NR. Could polygenic risk scores be useful in psychiatry?: a review. JAMA Psychiatry. 2021;78:210-9.

7. Benros ME, Mortensen PB. Role of infection, autoimmunity, atopic disorders, and the immune system in schizophrenia: evidence from epidemiological and genetic studies. Curr Top Behav Neurosci. 2020;44:141-59.

8. Rosenblat JD, Mclntyre RS. Bipolar disorder and immune dysfunction: epidemiological findings, proposed pathophysiology and clinical implications. Brain Sci. 2017;7:144.

9. Tylee DS, Sun J, Hess JL, Tahir MA, Sharma E, Malik R, et al. Genetic correlations among psychiatric and immune-related phenotypes based on genome-wide association data. Am J Med Genet B Neuropsychiatr Genet. 2018;177:641-57.

10. Khandaker GM, Zimbron J, Lewis G, Jones PB. Prenatal maternal infection, neurodevelopment and adult schizophrenia: a systematic review of populationbased studies. Psychol Med. 2013;43:239-57.

11. Benros ME, Nielsen PR, Nordentoft M, Eaton WW, Dalton SO, Mortensen PB. Autoimmune diseases and severe infections as risk factors for schizophrenia: a 30-year population-based register study. Am J Psychiatry. 2011;168:1303-10.

12. Benros ME, Waltoft BL, Nordentoft M, Ostergaard SD, Eaton WW, Krogh J, et al. Autoimmune diseases and severe infections as risk factors for mood disorders: a nationwide study. JAMA Psychiatry. 2013;70:812-20.

13. Pape K, Tamouza R, Leboyer M, Zipp F. Immunoneuropsychiatry-novel perspectives on brain disorders. Nat Rev Neurol. 2019;15:317-28.

14. Kroken RA, Sommer IE, Steen VM, Dieset I, Johnsen E. Constructing the immune signature of schizophrenia for clinical use and research; an integrative review translating descriptives into diagnostics. Front Psychiatry. 2018;9:753.
15. Luo $Y$, He H, Zhang $M$, Huang $X$, Fan N. Altered serum levels of TNF- $a$, IL- 6 and IL-18 in manic, depressive, mixed state of bipolar disorder patients. Psychiatry Res. 2016;244:19-23.

16. Baumeister D, Ciufolini S, Mondelli V. Effects of psychotropic drugs on inflammation: consequence or mediator of therapeutic effects in psychiatric treatment? Psychopharmacology. 2016;233:1575-89.

17. Jeppesen R, Benros ME. Autoimmune diseases and psychotic disorders. Front Psychiatry. 2019;10:131.

18. Germolec DR, Shipkowski KA, Frawley RP, Evans E. Markers of inflammation. Methods Mol Biol. 2018;1803:57-79.

19. Volin MV, Koch AE. Interleukin-18: a mediator of inflammation and angiogenesis in rheumatoid arthritis. J Interferon Cytokine Res. 2011;31:745-51.

20. Baert L, Manfroi B, Casez O, Sturm N, Huard B. The role of APRIL-a proliferation inducing ligand-in autoimmune diseases and expectations from its targeting. $J$ Autoimmun. 2018;95:179-90.

21. Mavropoulou E, Mechie NC, Knoop R, Petzold G, Ellenrieder V, Kunsch S, et al. Association of serum interleukin-6 and soluble interleukin-2-receptor levels with disease activity status in patients with inflammatory bowel disease: a prospective observational study. PLoS ONE. 2020;15:e0233811.

22. Migliorini P, Italiani P, Pratesi F, Puxeddu I, Boraschi D. The IL-1 family cytokines and receptors in autoimmune diseases. Autoimmun Rev. 2020;19:102617.

23. Smeland OB, Frei O, Fan CC, Shadrin A, Dale AM, Andreassen OA. The emerging pattern of shared polygenic architecture of psychiatric disorders, conceptual and methodological challenges. Psychiatr Genet. 2019;29:152-9.

24. Richards A, Horwood J, Boden J, Kennedy M, Sellers R, Riglin L, et al. Associations between schizophrenia genetic risk, anxiety disorders and manic/hypomanic episode in a longitudinal population cohort study. $\mathrm{Br} J$ Psychiatry. 2019;214:96-102.

25. Smoller JW, Kendler K, Craddock N. Identification of risk loci with shared effects on five major psychiatric disorders: a genome-wide analysis. Lancet. 2013;381:1371-9.

26. Duncan LE, Ratanatharathorn A, Aiello AE, Almli LM, Amstadter AB, Ashley-Koch $A E$, et al. Largest GWAS of PTSD ( $N=20070)$ yields genetic overlap with schizophrenia and sex differences in heritability. Mol Psychiatry. 2018;23:666-73.

27. O'Connell KS, Shadrin A, Bahrami S, Smeland OB, Bettella F, Frei O, et al. Identification of genetic overlap and novel risk loci for attention-deficit/hyperactivity disorder and bipolar disorder. Mol Psychiatry. 2019;26:4055-65.

28. Smeland OB, Bahrami S, Frei O, Shadrin A, O'Connell K, Savage J, et al. Genomewide analysis reveals extensive genetic overlap between schizophrenia, bipolar disorder, and intelligence. Mol Psychiatry. 2020;25:844-53.

29. Smeland OB, Wang Y, Lo MT, Li W, Frei O, Witoelar A, et al. Identification of genetic loci shared between schizophrenia and the big five personality traits. Sci Rep. 2017;7:2222.

30. Lo MT, Hinds DA, Tung JY, Franz C, Fan CC, Wang Y, et al. Genome-wide analyses for personality traits identify six genomic loci and show correlations with psychiatric disorders. Nat Genet. 2017:49:152-6.

31. Dowlati Y, Herrmann N, Swardfager W, Liu H, Sham L, Reim EK, et al. A metaanalysis of cytokines in major depression. Biol Psychiatry. 2010;67:446-57.

32. Dunn GA, Nigg JT, Sullivan EL. Neuroinflammation as a risk factor for attention deficit hyperactivity disorder. Pharm Biochem Behav. 2019;182:22-34.

33. Meltzer A, Van de Water J. The role of the immune system in autism spectrum disorder. Neuropsychopharmacology 2017;42:284-98.

34. Hori H, Kim Y. Inflammation and post-traumatic stress disorder. Psychiatry Clin Neurosci. 2019;73:143-53.

35. Bufalino $C$, Hepgul N, Aguglia E, Pariante $C M$. The role of immune genes in the association between depression and inflammation: a review of recent clinical studies. Brain Behav Immun. 2013;31:31-47.

36. Torres AR, Sweeten $T L$, Johnson RC, Odell D, Westover JB, Bray-Ward $P$, et al. Common genetic variants found in HLA and KIR immune genes in autism spectrum disorder. Front Neurosci. 2016;10:463.

37. Hope S, Hoseth E, Dieset I, Morch RH, Aas M, Aukrust P, et al. Inflammatory markers are associated with general cognitive abilities in schizophrenia and bipolar disorder patients and healthy controls. Schizophr Res. 2015;165:188-94.

38. Luchetti M, Barkley JM, Stephan Y, Terracciano A, Sutin AR. Five-factor model personality traits and inflammatory markers: new data and a meta-analysis. Psychoneuroendocrinology. 2014;50:181-93.

39. Ahola-Olli AV, Würtz P, Havulinna AS, Aalto K, Pitkänen N, Lehtimäki T, et al. Genome-wide association study identifies 27 loci influencing concentrations of circulating cytokines and growth factors. Am J Hum Genet. 2017;100:40-50.

40. Mistry S, Harrison JR, Smith DJ, Escott-Price V, Zammit S. The use of polygenic risk scores to identify phenotypes associated with genetic risk of bipolar disorder and depression: a systematic review. J Affect Disord. 2018;234:148-55.

41. Costello H, Gould RL, Abrol E, Howard R. Systematic review and meta-analysis of the association between peripheral inflammatory cytokines and generalised anxiety disorder. BMJ Open. 2019;9:e027925. 
42. Maurel $M$, Castagné $R$, Berger $E$, Bochud $M$, Chadeau-Hyam $M$, Fraga $S$, et al. Patterning of educational attainment across inflammatory markers: findings from a multi-cohort study. Brain Behav Immun. 2020;90:303-10.

43. Mørch RH, Dieset I, Færden A, Reponen EJ, Hope S, Hoseth EZ, et al. Inflammatory markers are altered in severe mental disorders independent of comorbid cardiometabolic disease risk factors. Psychol Med. 2019;49:1749-57.

44. Szabo A, O'Connell KS, Ueland T, Sheikh MA, Agartz I, Andreou D, et al. Increased circulating IL-18 levels in severe mental disorders indicate systemic inflammasome activation. Brain Behav Immun. 2022;99:299-306. https://doi.org/10.1016/ j.bbi.2021.10.017

45. Engh JA, Ueland T, Agartz I, Andreou D, Aukrust P, Boye B, et al. Plasma levels of the cytokines $B$ cell-activating factor (BAFF) and a proliferation-inducing ligand (APRIL) in schizophrenia, bipolar, and major depressive disorder: a cross sectional, multisite study. Schizophr Bull. 2022;48:37-46.

46. Sheikh M, Ueland T, O'Connell KS, Agartz I, Andreou D, Aukrust P, et al. Systemic cell-adhesion molecules (CAM) in severe mental illness-potential role of intracellular CAM-1 in linking peripheral and neuro-inflammation. 2022 (submitted).

47. Mørch RH, Dieset I, Faerden A, Hope S, Aas M, Nerhus M, et al. Persistent increase in TNF and IL-1 markers in severe mental disorders suggests trait-related inflammation: a one year follow-up study. Acta Psychiatr Scand. 2017;136:400-8.

48. Ventura J, Liberman RP, Green MF, Shaner A, Mintz J. Training and quality assurance with the structured clinical interview for DSM-IV (SCID-I/P). Psychiatry Res. 1998;79:163-73.

49. Loh PR, Danecek P, Palamara PF, Fuchsberger C, Reshef YA, Finucane HK, et al. Reference-based phasing using the Haplotype Reference Consortium panel. Nat Genet. 2016:48:1443-8.

50. Li Y, Willer CJ, Ding J, Scheet P, Abecasis GR. MaCH: using sequence and genotype data to estimate haplotypes and unobserved genotypes. Genet Epidemiol. 2010;34:816-34.

51. Das S, Forer L, Schonherr S, Sidore C, Locke AE, Kwong A, et al. Next-generation genotype imputation service and methods. Nat Genet. 2016;48:1284-7.

52. McCarthy S, Das S, Kretzschmar W, Delaneau O, Wood AR, Teumer A, et al. A reference panel of 64,976 haplotypes for genotype imputation. Nat Genet. 2016;48:1279-83.

53. Purcell SM, Wray NR, Stone JL, Visscher PM, O'Donovan MC, Sullivan PF, et al. Common polygenic variation contributes to risk of schizophrenia and bipolar disorder. Nature. 2009:460:748-52.

54. Choi SW, O'Reilly PF. PRSice-2: polygenic risk score software for biobank-scale data. Gigascience. 2019;8:giz082.

55. Okada Y, Wu D, Trynka G, Raj T, Terao C, Ikari K, et al. Genetics of rheumatoid arthritis contributes to biology and drug discovery. Nature. 2014;506:376-81.

56. Bentham J, Morris DL, Graham DSC, Pinder CL, Tombleson P, Behrens TW, et al. Genetic association analyses implicate aberrant regulation of innate and adaptive immunity genes in the pathogenesis of systemic lupus erythematosus. Nat Genet. 2015;47:1457-64.

57. de Lange KM, Moutsianas L, Lee JC, Lamb CA, Luo Y, Kennedy NA, et al. Genome-wide association study implicates immune activation of multiple integrin genes in inflammatory bowel disease. Nat Genet. 2017;49:256-61.

58. Ellinghaus D, Ellinghaus E, Nair RP, Stuart PE, Esko T, Metspalu A, et al. Combined analysis of genome-wide association studies for Crohn disease and psoriasis identifies seven shared susceptibility loci. Am J Hum Genet. 2012;90:636-47.

59. Dubois PC, Trynka G, Franke L, Hunt KA, Romanos J, Curtotti A, et al. Multiple common variants for celiac disease influencing immune gene expression. Nat Genet. 2010;42:295-302.

60. Barrett JC, Clayton DG, Concannon P, Akolkar B, Cooper JD, Erlich HA, et al. Genome-wide association study and meta-analysis find that over 40 loci affect risk of type 1 diabetes. Nat Genet. 2009:41:703-7.

61. Ligthart S, Vaez A, Võsa U, Stathopoulou MG, de Vries PS, Prins BP, et al. Genome analyses of $>200,000$ individuals identify 58 loci for chronic inflammation and highlight pathways that link inflammation and complex disorders. Am J Hum Genet. 2018;103:691-706.

62. Wray NR, Ripke S, Mattheisen M, Trzaskowski M, Byrne EM, Abdellaoui A, et al. Genome-wide association analyses identify 44 risk variants and refine the genetic architecture of major depression. Nat Genet. 2018;50:668-81.

63. Purves KL, Coleman JRI, Meier SM, Rayner C, Davis KAS, Cheesman R, et al. A major role for common genetic variation in anxiety disorders. Mol Psychiatry. 2020;25:3292-303.

64. Grove J, Ripke S, Als TD, Mattheisen M, Walters RK, Won H, et al. Identification of common genetic risk variants for autism spectrum disorder. Nat Genet. 2019;51:431-44.

65. Demontis D, Walters RK, Martin J, Mattheisen M, Als TD, Agerbo E, et al. Discovery of the first genome-wide significant risk loci for attention deficit/ hyperactivity disorder. Nat Genet. 2019;51:63-75.
66. Nievergelt CM, Maihofer AX, Klengel T, Atkinson EG, Chen CY, Choi KW, et al. International meta-analysis of PTSD genome-wide association studies identifies sex- and ancestry-specific genetic risk loci. Nat Commun. 2019;10:4558.

67. Savage JE, Jansen PR, Stringer S, Watanabe K, Bryois J, de Leeuw CA, et al. Genome-wide association meta-analysis in 269,867 individuals identifies new genetic and functional links to intelligence. Nat Genet. 2018;50:912-9.

68. Lee JJ, Wedow R, Okbay A, Kong E, Maghzian O, Zacher M, et al. Gene discovery and polygenic prediction from a genome-wide association study of educational attainment in 1.1 million individuals. Nat Genet. 2018;50:1112-21.

69. Müller $N$. The role of intercellular adhesion molecule-1 in the pathogenesis of psychiatric disorders. Front Pharm. 2019;10:1251.

70. George-Chandy A, Trysberg E, Eriksson K. Raised intrathecal levels of APRIL and BAFF in patients with systemic lupus erythematosus: relationship to neuropsychiatric symptoms. Arthritis Res Ther. 2008;10:R97.

71. Yuan N, Chen Y, Xia Y, Dai J, Liu C. Inflammation-related biomarkers in major psychiatric disorders: a cross-disorder assessment of reproducibility and specificity in 43 meta-analyses. Transl Psychiatry. 2019;9:233.

72. Berry CM, Gruys ML, Sackett PR. Educational attainment as a proxy for cognitive ability in selection: effects on levels of cognitive ability and adverse impact. J Appl Psychol. 2006;91:696-705.

73. Comes AL, Senner F, Budde M, Adorjan K, Anderson-Schmidt H, Andlauer TFM, et al. The genetic relationship between educational attainment and cognitive performance in major psychiatric disorders. Transl Psychiatry. 2019;9:210.

74. Lotrich FE, Butters MA, Aizenstein H, Marron MM, Reynolds CF 3rd, Gildengers AG. The relationship between interleukin-1 receptor antagonist and cognitive function in older adults with bipolar disorder. Int J Geriatr Psychiatry. 2014;29:635-44.

75. Bossù $P$, Ciaramella $A$, Salani $F$, Bizzoni $F$, Varsi $E$, Di lulio $F$, et al. Interleukin-18 produced by peripheral blood cells is increased in Alzheimer's disease and correlates with cognitive impairment. Brain Behav Immun. 2008;22:487-92.

76. Wu JQ, Chen DC, Tan YL, Tan SP, Xiu MH, Wang ZR, et al. Altered interleukin-18 levels are associated with cognitive impairment in chronic schizophrenia. J Psychiatr Res. 2016;76:9-15.

77. Iwata M, Ota KT, Duman RS. The inflammasome: pathways linking psychological stress, depression, and systemic illnesses. Brain Behav Immun. 2013;31:105-14.

78. Hylén U, Eklund D, Humble M, Bartoszek J, Särndahl E, Bejerot S. Increased inflammasome activity in markedly ill psychiatric patients: an explorative study. J Neuroimmunol. 2020;339:577119.

79. Rafnsson SB, Deary IJ, Smith FB, Whiteman MC, Rumley A, Lowe GD, et al. Cognitive decline and markers of inflammation and hemostasis: the Edinburgh Artery Study. J Am Geriatr Soc. 2007;55:700-7.

80. Hargreaves A, Anney R, O'Dushlaine C, Nicodemus KK, Gill M, Corvin A, et al. The one and the many: effects of the cell adhesion molecule pathway on neuropsychological function in psychosis. Psychol Med. 2014;44:2177-87.

81. Dowlatshahi EA, van der Voort EA, Arends LR, Nijsten T. Markers of systemic inflammation in psoriasis: a systematic review and meta-analysis. $\mathrm{Br} J$ Dermatol. 2013;169:266-82.

82. Ungprasert $\mathrm{P}$, Wijarnpreecha $\mathrm{K}$, Cheungpasitporn W. Patients with psoriasis have a higher risk of schizophrenia: a systematic review and meta-analysis of observational studies. J Postgrad Med. 2019;65:141-5.

83. Goodman WA, Levine AD, Massari JV, Sugiyama H, McCormick TS, Cooper KD. IL6 signaling in psoriasis prevents immune suppression by regulatory T cells. J Immunol. 2009;183:3170-6.

84. Pouget JG. The emerging immunogenetic architecture of schizophrenia. Schizophr Bull. 2018;44:993-1004.

85. Hall J. Schizophrenia-an anxiety disorder? Br J Psychiatry. 2017;211:262-3.

86. Vogelzangs $\mathrm{N}$, de Jonge $\mathrm{P}$, Smit JH, Bahn S, Penninx BW. Cytokine production capacity in depression and anxiety. Transl Psychiatry. 2016;6:e825.

87. Koh KB, Lee BK. Reduced lymphocyte proliferation and interleukin-2 production in anxiety disorders. Psychosom Med. 1998;60:479-83.

88. Tsai SY, Yang YY, Kuo CJ, Chen CC, Leu SJ. Effects of symptomatic severity on elevation of plasma soluble interleukin-2 receptor in bipolar mania. J Affect Disord. 2001;64:185-93.

89. Deyoung CG. The neuromodulator of exploration: a unifying theory of the role of dopamine in personality. Front Hum Neurosci. 2013;7:762.

90. Power RA, Steinberg S, Bjornsdottir G, Rietveld CA, Abdellaoui A, Nivard MM, et al. Polygenic risk scores for schizophrenia and bipolar disorder predict creativity. Nat Neurosci. 2015;18:953-5.

91. Shi J, Yao Y, Zhan C, Mao Z, Yin F, Zhao X. The relationship between big five personality traits and psychotic experience in a large non-clinical youth sample: the mediating role of emotion regulation. Front Psychiatry. 2018;9:648.

92. Rose AM, Bell LC. Epistasis and immunity: the role of genetic interactions in autoimmune diseases. Immunology. 2012;137:131-8. 
93. Girshkin L, Matheson SL, Shepherd AM, Green MJ. Morning cortisol levels in schizophrenia and bipolar disorder: a meta-analysis. Psychoneuroendocrinology. 2014;49:187-206.

94. Oswald LM, Zandi P, Nestadt G, Potash JB, Kalaydjian AE, Wand GS. Relationship between cortisol responses to stress and personality. Neuropsychopharmacology. 2006;31:1583-91.

95. Wedervang-Resell K, Friis S, Lonning V, Smelror RE, Johannessen C, Reponen EJ, et al. Increased interleukin 18 activity in adolescents with early-onset psychosis is associated with cortisol and depressive symptoms. Psychoneuroendocrinology. 2020;112:104513.

96. Simpson S, Kaislasuo J, Guller S, Pal L. Thermal stability of cytokines: a review. Cytokine. 2020;125:154829.

97. Binnington B, Sakac D, Yi Q, Tong TN, Parmar N, Duong TT, et al. Stability of 40 cytokines/chemokines in chronically ill patients under different storage conditions. Cytokine. 2020;130:155057.

98. Belvederi Murri M, Pariante CM, Dazzan P, Hepgul N, Papadopoulos AS, Zunszain $P$, et al. Hypothalamic-pituitary-adrenal axis and clinical symptoms in firstepisode psychosis. Psychoneuroendocrinology. 2012;37:629-44.

99. Kwon YC, Lim J, Bang SY, Ha E, Hwang MY, Yoon K, et al. Genome-wide association study in a Korean population identifies six novel susceptibility loci for rheumatoid arthritis. Ann Rheum Dis. 2020;79:1438-45.

100. Brodin P, Jojic V, Gao T, Bhattacharya S, Angel CJ, Furman D, et al. Variation in the human immune system is largely driven by non-heritable influences. Cell. 2015;160:37-47.

\section{ACKNOWLEDGEMENTS}

The authors thank the patients for participating in the study and contributing colleagues at NORMENT, especially the bio-psychiatry, genetic, biostatistical, clinical and database group. We would like to thank the research participants and employees of 23 andMe, Inc. for making this work possible.

\section{AUTHOR CONTRIBUTIONS}

MCFW and NES wrote the first draft of the paper. MCFW, KVW, SHL, LR, GH, MBEGO, $\mathrm{MH}, \mathrm{IA}, \mathrm{SD}, \mathrm{IM}, \mathrm{PA}, \mathrm{TU}, \mathrm{OAA}$, and NES recruited participants and gathered clinical data and blood samples or analyzed immune markers. NES, OAA, and KVW planned, supervised, and coordinated the work. MCFW, NES, OAA, KVW, and AS analyzed the data and interpreted the results. All authors contributed to and revised the article critically for important intellectual content and approved the final article. All authors are accountable for all aspects of the work

\section{FUNDING}

This work was supported by the Research Council of Norway (grant numbers 262656, 273291, 248778, and 223273); and the South-East Norway Regional Health Authority (grant numbers 2019-108 and 2017-112).

\section{COMPETING INTERESTS}

Author OAA has received Speaker's honorarium from Lundbeck, Sunovion, and is a consultant to HealthLytix. The remaining authors declare no competing interests.

\section{ADDITIONAL INFORMATION}

Supplementary information The online version contains supplementary material available at https://doi.org/10.1038/s41398-022-01811-6.

Correspondence and requests for materials should be addressed to Maren Caroline Frogner Werner.

Reprints and permission information is available at http://www.nature.com/ reprints

Publisher's note Springer Nature remains neutral with regard to jurisdictional claims in published maps and institutional affiliations.

Open Access This article is licensed under a Creative Commons At Atribution 4.0 International License, which permits use, sharing, adaptation, distribution and reproduction in any medium or format, as long as you give appropriate credit to the original author(s) and the source, provide a link to the Creative Commons license, and indicate if changes were made. The images or other third party material in this article are included in the article's Creative Commons license, unless indicated otherwise in a credit line to the material. If material is not included in the article's Creative Commons license and your intended use is not permitted by statutory regulation or exceeds the permitted use, you will need to obtain permission directly from the copyright holder. To view a copy of this license, visit http://creativecommons. org/licenses/by/4.0/

(c) The Author(s) 2022 